[white paper]

Diamond Open Access

\title{
Propriedades Reais: algoritmo de aprendizagem
}

\author{
Colaboração Matemática Aberta ${ }^{1}$
}

13 de Abril de 2022

\begin{abstract}
Resumo
Neste artigo apresentamos algumas propriedades dos números reais por meio de um algoritmo de aprendizagem.
\end{abstract}

palavras-chave: números reais, matemática, algoritmo de aprendizagem

A versão mais atualizada deste artigo está disponível em

https://osf.io/y452t/download

https: //zenodo.org/record/6367822

\section{Preâmbulo}

1. Ter ou não inclusão social (via escola/universidade) é uma decisão dos educadores e suas autoridades. (parafraseando Luckesi, 2018) [1]

\section{Introdução}

2. algoritmo = conjunto de diretrizes; receita; instruçôes

${ }^{1}$ Todos os autores com suas afiliações aparecem no final deste artigo. 
3. Um algoritmo de aprendizagem consiste em um material didáticopedagógico que permite compreender o ponto de partida e progredir a partir de pequenos saltos de aprendizagem [1].

4. O foco de um algoritmo de aprendizagem deve ser na aprendizagem necessária e no empreendimento de um conteúdo/habilidade por vez $[1,2]$.

5. Por aprendizagem necessária, queremos dizer ensinar o estritamente necessário, isto é, não precisa ensinar nem a mais, nem a menos.

6. pequenos saltos $=$ um conteúdo/habilidade por vez

\section{Pré-requisitos}

7. [3-5]

\section{Algoritmo de Aprendizagem}

8. Deixamos propositalmente espaços em branco neste white paper para que ele possa ser preenchido com anotações e cálculos extras durante seu estudo.

\section{Algumas definições}

9. Sejam $a, b, c \in \mathbb{R}$.

10. A palavra comutatividade está associada com comutar, que significa realizar troca ou permuta de.

11. Associatividade vem de associar; associe essa palavra com o símbolo de parêntesis ( ).

12. Justaposição: $a b$ é o mesmo que $a \cdot b$, que significa $a$ vezes $b$. 
13. É comum não escrevermos o símbolo de multiplicação $(\cdot)$ quando não causar ambiguidade.

14. Lembre-se que quando não aparece nada do lado esquerdo de um número real, na verdade temos

$$
2=+2=+1 \cdot 2 .
$$

15. O item (14) é para lembrar que nem sempre colocamos o sinal positivo à esquerda de um número e, também, que o número 1 está multiplicando todos os números já que essa multiplicação por 1 não altera o resultado final.

\section{Propriedades dos números reais}

16. Vamos começar com exemplos para prosseguir, então, com resultados mais gerais, isto é, na forma literal (usando letras).

17. Quando generalizamos um resultado utilizando letras quer dizer que ele é válido para qualquer valor de um dado conjunto.

18. Aqui estamos considerando o conjunto dos números reais.

\section{Comutatividade da Soma}

19. comutatividade da soma = trocar a ordem da soma

20. Exemplo

$$
\underbrace{2+3}_{5}=\underbrace{3+2}_{5}
$$

21. Caso geral $(a, b \in \mathbb{R})$

$$
a+b=b+a
$$

22. O resultado (21) vale, também, para números negativos. 
23.

$$
\underbrace{-4+5}_{1}=\underbrace{+5-4}_{1}
$$

24.

$$
\underbrace{-1-2}_{-3}=\underbrace{-2-1}_{-3}
$$

\section{Comutatividade do Produto}

25. comutatividade do produto = trocar a ordem da multiplicação 26.

$$
\underbrace{2 \cdot 3}_{6}=\underbrace{3 \cdot 2}_{6}
$$

27.

$$
a b=b a
$$

28. O resultado (27) vale, também, para números negativos.

29. Ao operar com números negativos, temos que colocar os parêntesis para indicarmos que se trata de uma multiplicação.

30.

$$
\underbrace{\underbrace{(-4)(+5)}_{(-) \cdot(+) \cdot 4 \cdot 5}}_{-20}=\underbrace{(+) \cdot(-) \cdot 5 \cdot 4}_{-20}
$$

31. Na multiplicação envolvendo números negativos, é interessante primeiro multiplicar os sinais, depois multiplicar os números "sem sinal",

$$
(-2) \cdot(+3)=(-) \cdot(+) \cdot 2 \cdot 3=-6 \text {. }
$$


32.

$$
\underbrace{(-1)(-2)}_{+2}=\underbrace{(-2)(-1)}_{+2}
$$

\section{Soma com números negativos}

33. Note que

$$
2-1=2+(-1),
$$

por isso a subtração pode ser vista como uma soma com um número negativo.

34. Quando a soma é feita com dois números negativos, mantém-se o sinal negativo,

$$
-1-2=-3 .
$$

35. Quando a soma é feita entre dois números com sinais opostos, mantémse o sinal do maior número.

36. Neste caso é mais fácil escrever primeiro o número positivo e depois o negativo, antes de concluir o cálculo,

$$
-1+2=2-1=1 .
$$

37.

$$
1-2=-1
$$

38.

$$
\forall a, b \in \mathbb{R}:-a+b=b-a
$$

39. Mostre que, dado $a=-3$,

$$
-a=+3 .
$$




\section{Produto com números negativos}

40. Use a regra:

Primeiro multiplique os sinais, depois multiplique os números.

41. Existem apenas dois resultados envolvendo o produto de sinais.

42. $(+) \cdot(+)=(-) \cdot(-)=+$

43. $(+) \cdot(-)=(-) \cdot(+)=-$

44. Em resumo, o produto de sinais iguais é positivo, enquanto que o produto de sinais opostos é negativo.

45. No exemplo a seguir, utilizamos a regra (40),

$$
-2 \cdot 3=(-2) \cdot(+3)=(-) \cdot(+) \cdot 2 \cdot 3=-6 .
$$

\section{Associatividade da Soma}

46. associatividade $\sim>$ parêntesis

47.

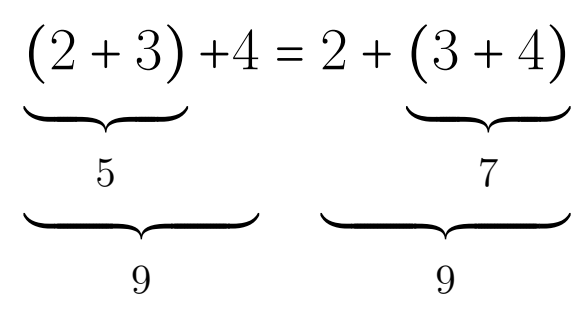

48. A fórmula geral é dada por

$$
\forall a, b, c \in \mathbb{R}:(a+b)+c=a+(b+c) .
$$

49. Para $a=-1, b=2$ e $c=-3$, temos que
(a) $(a+b)+c=(-1+2)+-3=1-3=-2$,
(b) $a+(b+c)=-1+(2+-3)=-1+(2-3)=-1+(-1)=-1-1=-2$, 
(c) portanto, o resultado é o mesmo, independente da ordem da soma.

50. Mostre que a fórmula (48) é válida para os seguintes casos.

51. $a=-2, \quad b=-3, \quad c=-4$

52. $a=-2, \quad b=3, \quad c=-4$

53. $a=2, \quad b=-3, \quad c=-4$ 


\section{Associatividade do Produto}

54.

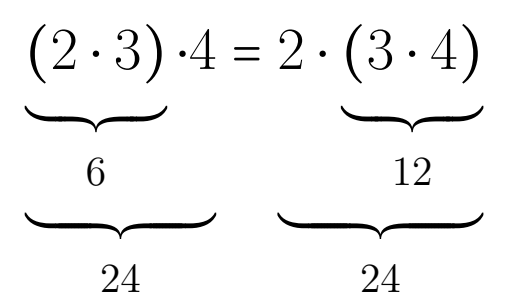

55.

$$
\forall a, b, c \in \mathbb{R}:(a b) c=a(b c)
$$

56. Mostre que a fórmula (55) é válida para os seguintes casos.

57. $a=-2, \quad b=-3, \quad c=-4$

58. $a=-2, \quad b=3, \quad c=-4$ 
59. $a=2, \quad b=-3, \quad c=-4$

\section{Distributiva}

60.

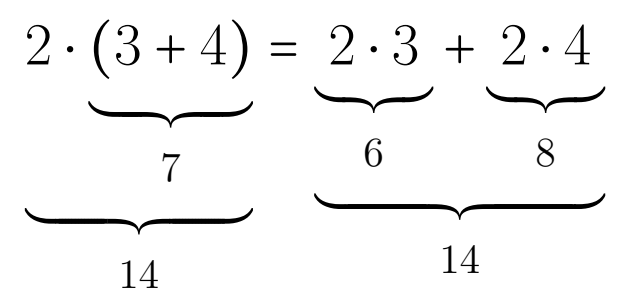

61. Distributiva pela esquerda

$$
\forall a, b, c \in \mathbb{R}: a \cdot(b+c)=a \cdot b+a \cdot c=a b+a c
$$

62. Distributiva pela direita

$$
\forall a, b, c \in \mathbb{R}:(b+c) \cdot a=b \cdot a+c \cdot a=b a+c a=a b+a c
$$

63. Note que na última igualdade de (62), utilizamos a comutatividade do produto.

64. Mostre, por meio da fórmula (61) ou (62), que a distributiva é válida para os seguintes casos. 
65. $a=-2, \quad b=-3, \quad c=-4$

66. $a=-2, \quad b=3, \quad c=-4$

67. $a=2, \quad b=-3, \quad c=-4$ 


\section{Resumo}

68. A ordem da soma e do produto entre dois números reais não altera o resultado.

69. O produto de sinais iguais é positivo, enquanto que o produto de sinais opostos é negativo.

70. comutatividade $\sim>$ comutar $\sim>$ trocar a ordem

71. associatividade $\sim>$ associar $\sim>$ com parêntesis

72. $a=+1 \cdot a$

73. $a+b=b+a$

74. $a b=b a$

75. $(+) \cdot(+)=(-) \cdot(-)=+$

76. $(+) \cdot(-)=(-) \cdot(+)=-$

77. $(a+b)+c=a+(b+c)$

78. $(a b) c=a(b c)$

79. $a \cdot(b+c)=a \cdot b+a \cdot c$

80. $(b+c) \cdot a=b a+c a$ 


\section{Ciência Aberta}

O arquivo latex para este artigo, juntamente com outros arquivos suplementares, estão disponíveis em [6,7]. Seja coautor(a) deste artigo, envie sua contribuição para mplobo@uft. edu.br.

\section{Consentimento}

O autor concorda com [8].

\section{Como citar este artigo?}

https://doi.org/10.31219/osf .io/y452t

https://zenodo.org/record/6367822

\section{Licença}

CC-By Attribution 4.0 International [9]

\section{Referências}

[1] Luckesi, Cipriano C. "Oficina sobre avaliação da aprendizagem no Ensino Superior". Universidade Federal do Tocantins, Araguaína, 2018.

[2] Lobo, Matheus P. "Matemática Minimalista: Menos É Mais." OSF Preprints, 18 Oct. 2020.

https://doi.org/10.31219/osf .io/pey6z

[3] OJMP Brasil. "Fundamentos da Matemática".

https://ojmpbr. wordpress. com/fundamentos-da-matematica

[4] Lobo, Matheus P. "Matemática Zero." OSF Preprints, 1 Oct. 2020. https://doi.org/10.31219/osf.io/dgsf2 
[5] Lobo, Matheus P. "Para Que Servem Os Números?." OSF Preprints, 8 Oct. 2020. https://doi.org/10.31219/osf.io/tyn7k

[6] Lobo, Matheus P. "Open Journal of Mathematics and Physics (OJMP)." OSF, 21 Apr. 2020.

https://doi.org/10.17605/osf .io/6hzyp

[7] https://zenodo.org/record/6367822

[8] Lobo, Matheus P. "Simple Guidelines for Authors: Open Journal of Mathematics and Physics." OSF Preprints, 15 Nov. 2019.

https://doi.org/10.31219/osf .io/fk836

[9] CC. Creative Commons. Attribution 4.0 International (CC BY 4.0) https://creativecommons.org/licenses/by/4.0

\section{Colaboração Matemática Aberta}

Matheus Pereira Lobo (autor principal, mplobo@uft.edu.br) $)^{1,2}$ https://orcid.org/0000-0003-4554-1372

${ }^{1}$ Universidade Federal do Tocantins (Brasil)

${ }^{2}$ Universidade Aberta (UAb, Portugal) 